\title{
Research on dynamic energy saving path planning algorithm based on real-time traffic information
}

\author{
Lin Liu ${ }^{1,}$, Yibin Zhang ${ }^{2, ~ b}$, Huizong Feng ${ }^{3, c}$ \\ ${ }^{1}$ School of Automation, Chongqing University of Posts and Telecommunications, ChongQing \\ 400065, China; \\ ${ }^{2}$ School of Automation, Chongqing University of Posts and Telecommunications, ChongQing \\ 400065, China; \\ ${ }^{3}$ School of Automation, Chongqing University of Posts and Telecommunications, ChongQing \\ 400065, China; \\ aliulin@cqupt.edu.cn, b304385733@qq.com, cfenghz@cqupt.edu.cn
}

Keywords: path planning, energy saving path, real-time traffic information, Dijkstra algorithm

\begin{abstract}
Path planning is an important way to alleviate traffic congestion, but the traditional path planning algorithm can only tell us the shortest distance path. Meanwhile, the traffic information is dynamic and changes over time, so the initial optimal path is likely to be invalid when traffic conditions change. A dynamic energy saving path planning algorithm (DESPP) based on real-time traffic information is proposed in this paper, which can plan the energy saving path for driver dynamically. A dynamic road network model is designed to reflect the actual traffic condition, and then an extended Dijkstra algorithm is elaborated, by setting the fuel consumption for the calculation of weight, changing the storage structure of road data, and using the improved quick sort algorithm to sort the weights, so that fast search to the adjacent node can be realized, then the dynamic energy saving path planning algorithm (DESPP) will be obtained. The simulation results show that DESPP algorithm is able to get the energy saving path correctly and effectively. It reduces about $15.19 \%$ of the total fuel consumption compared with the traditional Dijkstra algorithm. The DESPP algorithm can be applied in finding energy saving path for navigation system, which is more suitable for the actual need of drivers.
\end{abstract}

\section{Introduction}

With the quick development of urban traffic, traffic congestion has become a serious social problem, which directly leads to increasing fuel consumption and serious environmental pollution. Path planning is to find a path from the starting point to the terminal point in a particular network according to some evaluation standards (such as the shortest or the fastest, etc), which is an important way to solve traffic congestion and save fuel consumption. Traditional path planning algorithm can only solve the static shortest path problem[1,2], due to not considering the real-time road conditions such as traffic jams, accidents, etc., the shortest path is not the optimal path. Therefore, many scholars have carried out extensive research on the path planning problem[3 6], and made some progress, but mainly focused on solving the shortest path or the shortest time, the research on energy saving path planning is very little. With the consideration of reducing fuel consumption, this paper based on Internet of Vehicles (IoVs) environment, access to the real-time traffic information, changes the traditional problem that find the shortest path into find the most efficient path. Taking into account the two conditions of the vehicle: Travel on the road segments and delay at intersections, a road network model is designed to reflect actual traffic state, we improved the traditional Dijkstra algorithm, and proposed the dynamic energy saving path planning algorithm(DESPP), simulation experiment is designed to verify the effectiveness of the algorithm. 


\section{Corresponding road network model}

A new dynamic road network model is designed in order to get a more accurate planning results, the details are as follows: Let $G=(V, E, T, L, R, D, V S)$ be a stochastic dynamic network. is the set of nodes; is the set of edges; is the set of time intervals. is the set of edge length; we denote the set of road impedance function by, function is road impedance function of edge. For the sake of simplicity, assume that the road impedance is constant when the vehicle is running on the road, the greater the value of the $r$, said the road congestion, the road speed is small, when the road is smooth, $\mathrm{r}=1$. is the set of intersection delay times. means the delay when vehicle through the intersection drive from to the road segment at time zone $t$. VS is the average speed of the edge, assume that the vehicle is traveling at the average speed of the edge. is the maximum speed.

Based on the above road network model, the real-time traffic data update only needs to transmit the road impedance, and the traffic state of the road network can be obtained, which can reduce the amount of data communication between the vehicle terminal and server.

\section{Algorithm design}

In this paper, the dynamic energy saving path planning problem is resolved based on real time traffic information. The corresponding algorithm is designed by modifying the calculation weight of traditional Dijkstra algorithm.

Set fuel consumption as the calculation weight. In this paper, the fuel consumption is used as the weight. Comprehensive consideration of the two main aspects: Vehicles running on the road segment and idling at the intersection. Assume that the vehicle is traveling at the average speed of the road segment, the amount of fuel consumed in road segment $\langle i, j\rangle$ can be calculated by its length and the and the oil consumption per kilometer, which is a function of its average driving speed for time zone $t$, as the shown in Fig.1[7]. The idling fuel consumption at the intersection of the vehicle can be calculated by idle time and idle fuel consumption rate.

The fuel consumption in road segment:

Where is the length of road segment, is the average speed of road segment at time zone $t$.

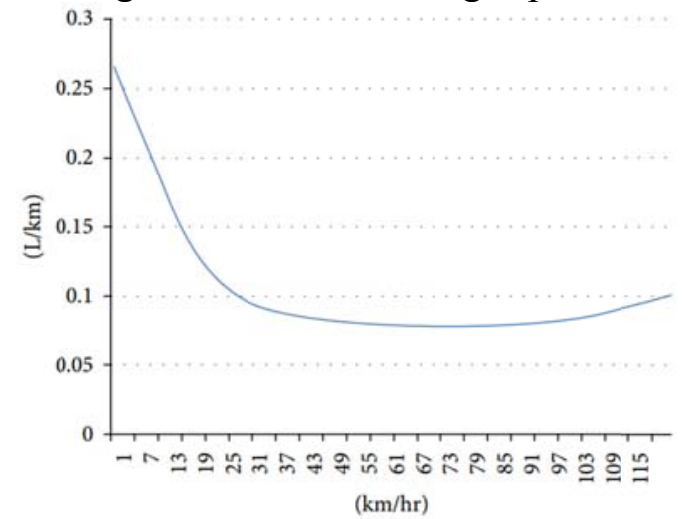

Fig. 1 An example of the relationship between the driving speed and the corresponding fuel consumption per kilometer.

Idle fuel consumption at the intersection $i$ is

Where is the time delay of the intersection $i$; is the idle fuel consumption rate of the vehicle, it can be measured experimentally.

At time zone $t$, the total fuel consumption of vehicle passing through road segment is

Obviously, by using the fuel consumption as the weight can meet the consistency principle.

Storage of road network topology relation. Traditional Dijkstra algorithm uses adjacency matrix store road network's topology. For the urban road network, the nodes is huge, it needs to open $\mathrm{N} \times \mathrm{N}$ ( $\mathrm{N}$ is node number) the storage space. The space complexity is $\mathrm{O}\left(\mathrm{N}^{2}\right)$. There will be a 
large number of 0 and elements in the adjacency matrix, computational efficiency and storage efficiency will be affected. Thus, we uses the adjacency list to store the network topology, at the same time, use an auxiliary bidirectional circulation linked list to store the adjacent node that remains to be sorted.

Fast realization of weight sorting. The key of Dijkstra algorithm is to select the smallest weight segmental arc from the nodes. In the traditional Dijkstra algorithm, adjacency node disorderly storage in an unordered list, if the weight of its adjacent node is not made any treatment, it needs to scan cycle several times to get the minimum weight arcs, this will reduce the efficiency of the algorithm. If the related adjacent node are sorted according to the weight, we can obtain the node of conform requirements in one cycle, which can improve the efficiency of the algorithm. In this paper, the weights are sorted by the improved quick sorting algorithm, then the optimization of related adjacent node is achieved, and the search time of the algorithm is reduced.

The basic idea of improved quick sort algorithm: supposed to sort the weight array is $W[0] \cdots \cdots$ $W[n-1]$, the mean value of the maximum element and the smallest element of the array to be sorted is Ave. Set Ave as the middle element, $k$ and $l$ are the first and last elements, $k=0, l=N-1$. Search from $l$ to the front $(l=l-1)$, find the first value $W[l]$ that less than Ave, meanwhile, search from $k$ to the back $(k=k+1)$, find the first value $W[k]$ that greater than Ave, exchange value of $W[l]$ and $W[k]$. Repeat the above steps until $\mathrm{k}=1$. Then, use recursive method to quickly sort the sub sequences, when the length of the array is less than a certain value, the recursion is stopped. Although such a quick sort after the end did not achieve the desired goal, but it has a certain initial sequence, can be used direct insertion sort algorithm. At the same time, using the method of set up signs to have ordered or is the same data sorting judgments. If in the process of scanning, there is no exchange of data occurred, indicating that the sequence has been ordered, so that the completion of the order.

Based on the above, the improve steps are as follows: Let $Z$ be the permanent mark node collection, $M$ is the adjacency node collection of the nodes that has marked; $N$ stores the sorting nodes; dist $[t]$ is the shortest path length from source $s$ to the node $t ;$ is the shortest path's forerunner node from $s$ to the $t ; d_{i j}$ is the distance from node $i$ to node $j$.

1) Initialization. $Z=\{s\}, N=M[s]$, , otherwise.

2) The node in $M[s]$ stores $N[]$ and carry on sorting, takes the heap top node $p$, if, then .

3) Renew the adjacency node collection near $p, N[]=M[p]-Z$; , if , then the forerunner node of is $p$.

4) Search $M[Z]-Z$, and store in $N[]$, designate the minimum value of $\operatorname{dist}[t]$ in $M[Z]-Z$, and store in $Z$, .

5) If all nodes have marked, then the algorithm terminate, otherwise changes over to the second step and continues to carry out, until all nodes gather in $Z$.

Based on the above content, the dynamic energy saving path planning algorithm (DESPP) based on real-time traffic information is obtained, as shown in Fig.2.

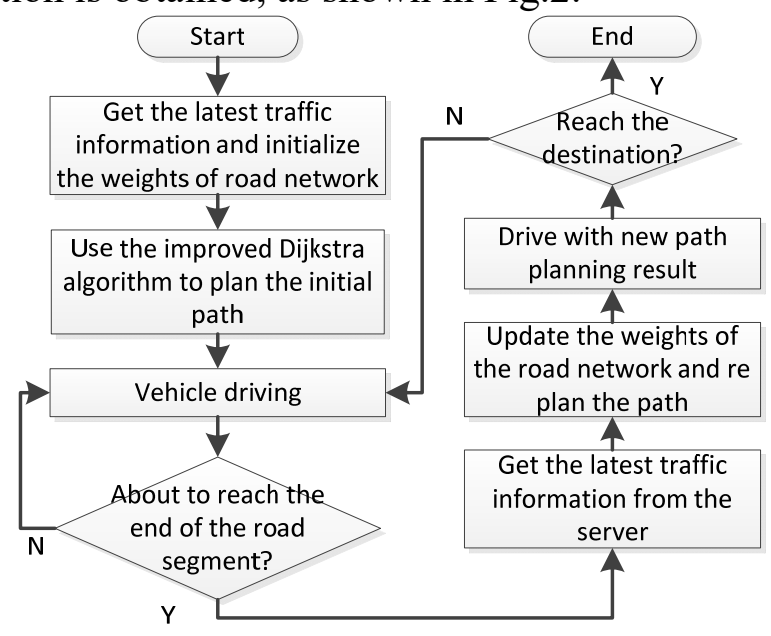

Fig. 2 The flow chart of dynamic energy saving path planning algorithm 


\section{Simulation experiment}

Experimental design. The experimental hardware environment: Lenovo Core(TM) i5-4590, $3.30 \mathrm{GHz}, \mathrm{RAM}$ capacity 4GB, Windows10 64 bit operating system. All programs are written in Java, the Java virtual machine version is JDK1.8.

In order to verify the correctness and validity of the algorithm, according to a city road network, a part of the road network is selected and simplified, which contains 72 nodes, 249 edges. The dynamic road network structure diagram is shown in Fig.3. Due to the limitation of the experimental environment, the real-time traffic information is generated by the program simulation, the number of time interval is set as $500, \mathrm{~T}=\{1,2, \ldots, 500\}$, and each time interval is $30 \mathrm{~s}$. Considering that the road segment is stable in most time, we only choose 100 intervals to change the road impedance. Meanwhile, the road resistance coefficient is $1 \%$ chance becomes infinite, indicating that this road impassable. Set the maximum speed of the road network is $70 \mathrm{~km} / \mathrm{h}$, the idle fuel consumption rate of vehicle is $0.5 \mathrm{~L} / \mathrm{h}$, and the intersection delay time is $5 \mathrm{~s} .30$ random node pairs are selected to carry out the experiment, in order to make the experimental results more scientific, we will keep the change process of traffic information in the files, after each test road network will be in accordance with the same process changes.

Simulation Results. We compare the effect of traditional Dijkstra (TD) algorithm and the dynamic energy saving path planning (DESPP) algorithm by fuel consumption and travel distance. Fig. 4 shows that, compared with the traditional path planning algorithm, the DESPP algorithm can effectively reduce the fuel consumption of vehicles. In the 30 groups, 28 groups of vehicle fuel consumption were significantly reduced, after the use of DESPP algorithm, the total fuel consumption savings of $15.19 \%$. Meanwhile, it is found that the group 15 and the group 27 are not reduced, but increased, this is because the DESPP algorithm can only obtain the optimal results at the present time. For global terms, according to the real-time traffic information frequently changing route leads to the increase of the distance of the vehicle, as shown in Fig.5, after adopting DESPP algorithm, the total distance of the vehicle increased $7.44 \%$ than traditional Dijkstra algorithm, there may be a case of fuel consumption does not fall, the solution to this problem is to predict the road conditions in a short time, which can be used as the next step in the research work. Overall, the experimental results show that after adopting DESPP algorithm, 93.3\% of vehicle driving fuel consumption decreased, this shows that the path planning method proposed in this article is feasible, which can effectively reduce the waste of fuel consumption and pollution emissions, and is more suitable for the actual needs of users.

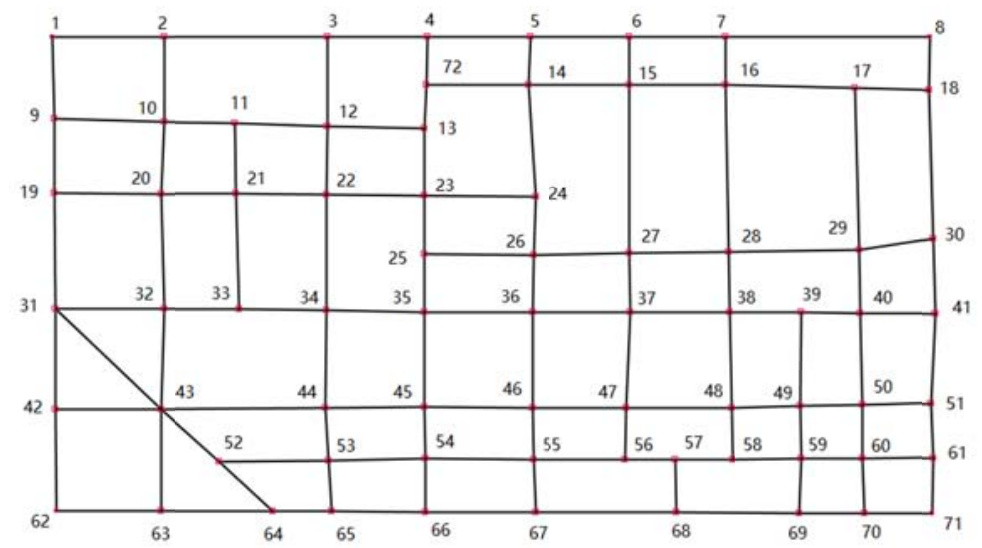

Fig. 3 The dynamic road network structure 


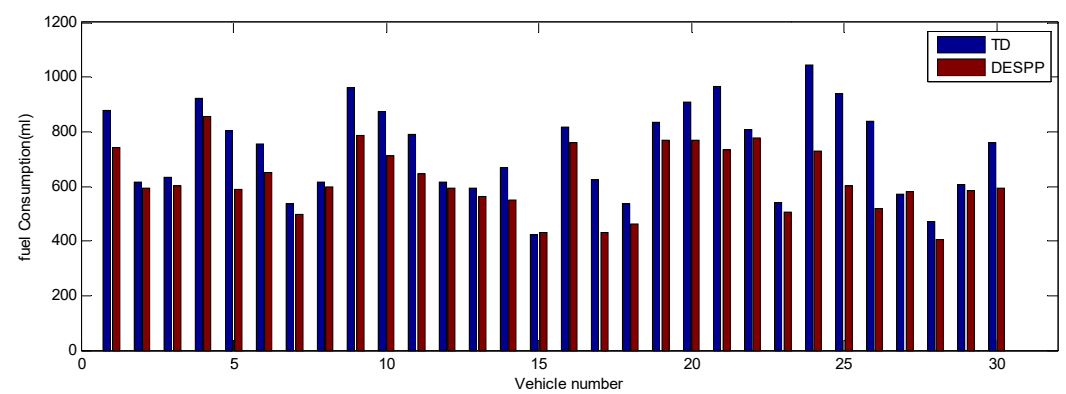

Fig. 4 The fuel consumption using TD algorithm vs. DESPP algorithm

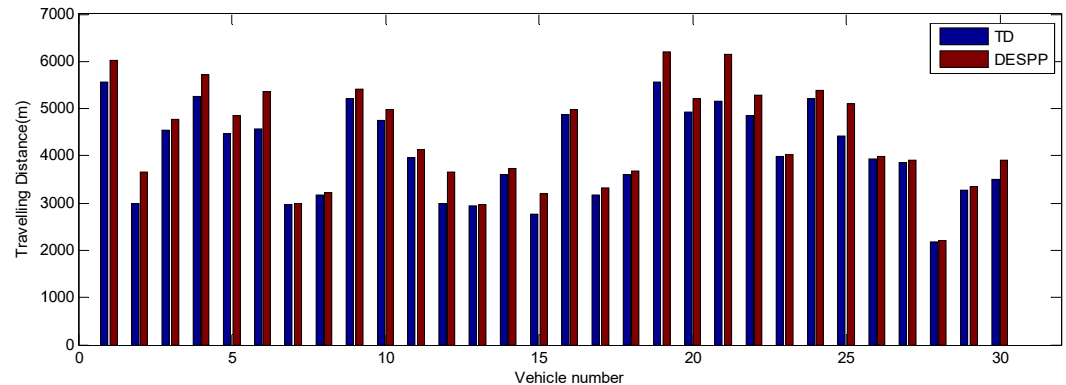

Fig. 5 The travelling distance using TD algorithm vs. DESPP algorithm

Application implementation. The above algorithm is implemented in IoVs platform. The dynamic energy saving navigation app is designed and implemented on the Google Android 4.1 operation system by using the open source map OpenStreetMap, which a regional map of Chongqing, China is selected. After setting the start point and end point, then the initial path is obtained, the vehicle drive on this path, as shown in Fig.6. During the driving process, the vehicle receives the real-time traffic information, and the current position is the start point, then re plan path, as shown in Fig.7.
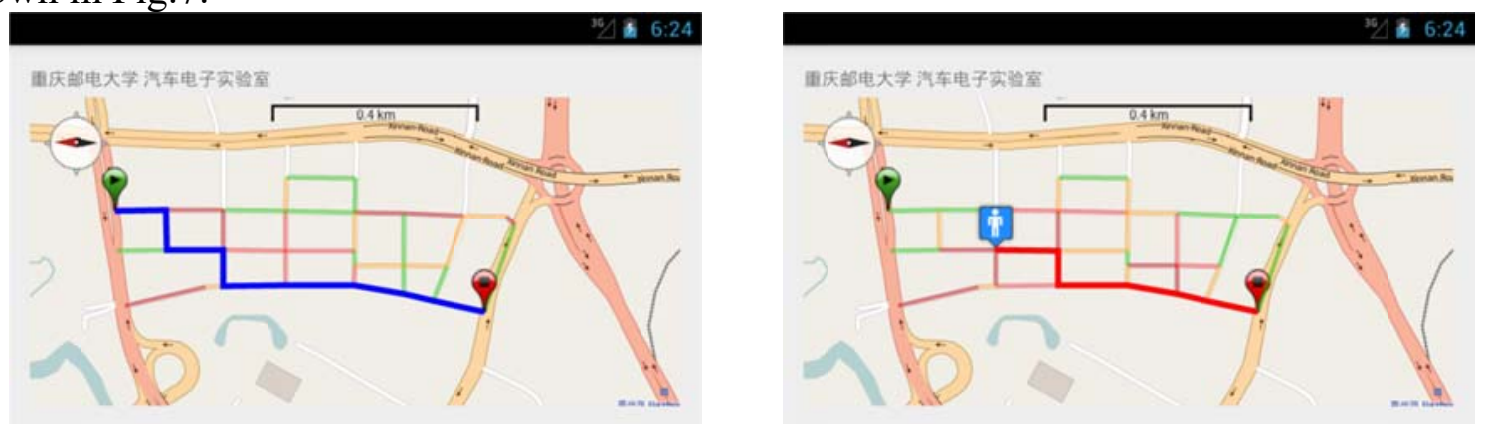

Fig. 6 Initial plan path Fig. 7 Replan path after receiving the traffic information

\section{Summary}

This paper presents a dynamic energy saving path planning algorithm based on real-time traffic information, a dynamic road network model is designed to reflect the actual traffic situation, and the traditional Dijkstra algorithm is improved. The experimental results show that the proposed algorithm can provide a more energy efficient driving route, and effectively reduce the fuel consumption.

\section{Acknowledgments}

This work was supported by Achievement Transfer Program of Institutions of Higher Education in Chongqing (KJZH14207) and Students Research and Training Program(SRTP)( A2015-78).

\section{References}

[1] SUN Li-jun, HU Xiang-pei, WANG Zheng. Reviews on Vehicle Routing Problem and Its 
Solution Methods[J]. Systems Engineering, 2006, 24(11):31-37.

[2] Dijkstra E W. A Note on Two Problems in Connection with Graphs[J]. Numerische Mathematics, 1959, 1(1):269--271.

[3] MA Renli, GUAN Zhengxi. Summation for Present Situation and Future Development of Path Planning Technology[J]. Modern Machinery, 2008(3):22-24.

[4] XIANG Dong-mei, CHEN Shu-hui. Research on the Shortest Time Path Planning Based on Dynamic Traffic[J]. Microcomputer Information, 2012,28(9):317-319.

[5] Chu K, Kim J, Jo K, et al. Real-time path planning of autonomous vehicles for unstructured road navigation[J]. International Journal of Automotive Technology, 2015, 16(4):653-668.

[6] Kong X, Yang J. Path Planning with Information on Travel Time Reliability[C]// Cota International Conference of Transportation Professionals. 2016.

[7] Chang I C, Tai H T, Yeh F H, et al. A VANET-Based Route Planning Algorithm for Travelling Time- and Energy-Efficient GPS Navigation App[J]. International Journal of Distributed Sensor Networks, 2013, 9(3):310-325. 